\title{
Two-Dimensional Coherent Charge Transport in Highly Ordered Conducting Polymers Doped by Solid State Diffusion
}

Keehoon Kang*, ${ }^{1}$ Shun Watanabe ${ }^{\dagger}{ }^{1,2,3}$ Katharina Broch, ${ }^{1}$ Alessandro Sepe, ${ }^{4}$ Adam Brown, ${ }^{1}$ Iyad Nasrallah, ${ }^{1}$ Mark Nikolka, ${ }^{1}$ Zhuping Fei, ${ }^{5}$ Martin Heeney, ${ }^{5}$ Daisuke Matsumoto, ${ }^{6}$ Kazuhiro Marumoto, ${ }^{6,7}$

Hisaaki Tanaka, ${ }^{8}$ Shin-ichi Kuroda, ${ }^{8}$ and Henning Sirringhaus ${ }^{\ddagger 1}$

${ }^{1}$ Cavendish Laboratory, University of Cambridge,

J. J. Thomson Avenue, Cambridge CB3 OHE, United Kingdom

${ }^{2}$ Department of Advanced Materials Science,

Graduate School of Frontier Sciences, The University of Tokyo,

5-1-5 Kashiwanoha, Kashiwa 277-8561, Chiba, Japan

${ }^{3}$ JST, PRESTO, 4-1-8 Honcho, Kawaguchi, Saitama, 332-0012, Japan

${ }_{4}^{4}$ Adolphe Merkle Institute, University of Fribourg,

Chemin des Verdiers 4 CH-1700 Fribourg Switzerland

${ }^{5}$ Department of Chemistry and Centre for Plastic Electronics,

Imperial College, London, SW7 2AZ, United Kingdom

${ }^{6}$ Division of Materials Science, University of Tsukuba, Tsukuba, Ibaraki 305-8573, Japan

${ }^{7}$ Tsukuba Research Center for Interdisciplinary Materials Science (TIMS),

University of Tsukuba, Tsukuba, Ibaraki 305-8571, Japan

${ }^{8}$ Department of Applied Physics, Nagoya University, Chikusa, Nagoya, 464-8603, Japan

\footnotetext{
* These authors contributed equally to this work

$\dagger$ These authors contributed equally to this work

$\ddagger$ Correspondence and requests for materials should be addressed to hs220@cam.ac.uk
} 
Doping is one of the most important methods to control charge carrier concentration in semiconductors. Ideally, the introduction of dopants should not perturb the ordered microstructure of the semiconducting host. In some systems, such as modulation-doped inorganic semiconductors or molecular charge transfer crystals, this can be achieved by spatially separating the dopants from the charge transport pathways. However, in conducting polymers dopants tend to be randomly distributed within the conjugated polymer and as a result the transport properties are strongly affected by the resulting structural and electronic disorder. Here, we show that in the highly ordered lamellar microstructure of a regioregular thiophene-based conjugated polymer a small-molecule p-type dopant can be incorporated by solid-state diffusion into the layers of solubilising side chains without disrupting the conjugated layers. In contrast to more disordered systems, this allows us to observe coherent, free-electron-like charge transport properties, including a nearly ideal Hall effect in a wide temperature range, a positive magneto-conductance due to weak localisation and Pauli paramagnetic spin susceptibility.

Conjugated small molecules and polymers continue to be researched intensively as semiconducting materials enabling a new generation of flexible, large-area optoelectronic devices. A common approach to p-type (n-type) doping is to co-deposit an organic electron acceptor (donor) with a suitable electron donating (accepting) host material. ${ }^{1-4}$ This leads to formation of charge transfer complexes (CTCs) in which an electronic charge is transferred between donor and acceptor. ${ }^{1,5}$ Unlike in organic charge transfer salts, which form separate crystalline donor and acceptor stacks, ${ }^{6}$ the incorporation of small molecule dopants into conjugated polymers is usually random and distorts the desired highly-ordered microstructure of the host. Although high conductivities can be achieved in conducting polymers, such as $\sigma \sim 10^{5} \mathrm{~S} \mathrm{~cm}^{-1}$ in a solid pellet of polyacetylene ${ }^{7}$ and of $\sigma \sim 2,000 \mathrm{~S}$ $\mathrm{cm}^{-1}$ in solution-processable polythiophenes, ${ }^{8}$ the charge transport mechanism in such disordered materials is complicated as a result of the structural and ensuing energetic disorder. ${ }^{9}$

In the last two decades, doped $\pi$-conjugated polymers have revealed metallic transport signatures, including observation of a negative temperature coefficient of conductivity 
$(d \sigma / d T<0),{ }^{10,11}$ Drude-like optical absorption ${ }^{12}$ and temperature-independent spin susceptibility. ${ }^{13}$ However, these observations have been difficult to reconcile with other transport coefficients, such as Hall effect measurements, which have provided much insight into the transport physics of organic semiconductor field-effect transistors. ${ }^{14-20}$ The Hall voltage, $V_{\mathrm{H}}$, measures the transverse electromotive force generated in a conductor when placed in a magnetic field $B$ oriented perpendicular to the current $I$ and arises in a semiclassical Boltzmann transport regime as a result of the Lorentz force that the coherent wavepacket of a delocalised electron with a defined wavevector experiences in the magnetic field. In this so-called ideal Hall effect regime, the Hall coefficient, $R_{\mathrm{H}}=\frac{V_{\mathrm{H}} t}{I B}$, is given by $R_{\mathrm{H}}=(e n)^{-1}$, where $n$ and $t$ are the carrier concentration and film thickness. In contrast, in a conduction regime dominated by hopping between localised states Hall voltages tend to be much smaller and also exhibit sign anomalies. ${ }^{21,22}$ Thereby, the observation of an ideal Hall effect can be interpreted as demonstration of coherent charge transport. However, Hall effect measurements in conjugated polymers have commonly yielded much smaller Hall coefficient than expected from the charge concentration, ${ }^{23,24}$ except for a specially prepared polyaniline. ${ }^{10}$ This presumably reflects the role of disorder and ensuing carrier localisation in most highly doped systems.

In addition to conventional chemical and electrochemical doping methods based on redox or acid-base reactions, ${ }^{25}$ controllable p-type doping has been achieved by co-depositing solution-processable $\pi$-conjugated polymers with a strong molecular acceptor, such as 2,3,5,6-tetrafluoro-7,7,8,8-tetracyanoquinodimethane $\left(\mathrm{F}_{4}\right.$-TCNQ). ${ }^{26,27}$ In a recent study doping of a thiophene-based $\pi$-conjugated polymer, poly (2,5-bis (3-hexadecylthiophen-2-yl) thieno [3,2-b] thiophene) (PBTTT; shown in Fig. 1a) by $\mathrm{F}_{4}$-TCNQ was investigated. ${ }^{28}$ The dopant was co-deposited with the polymer from solution and found to be intercalated between the $\pi$ stacked conjugated chains, presumably introducing some disorder into the $\pi$-stacking; the resulting electrical conductivity at the maximum achievable doping level was relatively moderate, i.e. only $2 \mathrm{~S} \mathrm{~cm}^{-1}$. Here, we study the same materials system, but we evaporate the $\mathrm{F}_{4}$-TCNQ molecule on top of a PBTTT layer that is pre-deposited via spin coating and allow the molecule to diffuse into the film. This results in the polymer retaining its highly ordered lamellar microstructure with the dopant incorporated into the layer of side chains. With this solid-state diffusion method we achieve a much higher electrical 
conductivity up to $248 \mathrm{~S} \mathrm{~cm}^{-1}$. We compare the transport properties of these films to those of amorphous poly(3,4-ethylenedioxythiophene): polystyrene sulfonate (PEDOT:PSS) to reveal structural order as a crucial element for charge coherence in conducting polymers.

To assess the extent of doping in our highly-conductive films obtained by thermally evaporating $\mathrm{F}_{4}$-TCNQ directly on top of the spin-coated PBTTT layer (Supplementary Section A) we performed UV-Vis absorption spectroscopy (Fig. 1b). When the polymer film is doped, the absorption centred around $555 \mathrm{~nm}(2.2 \mathrm{eV})$, which corresponds to a neutral absorption peak, is bleached and sub-band gap transitions appear around $830 \mathrm{~nm}$ which correspond to a broad polaron absorption of PBTTT. The peaks at $767 \mathrm{~nm}$ and $869 \mathrm{~nm}$ indicate the presence of fully ionised singly-charged $\mathrm{F}_{4}$-TCNQ anions on top of the broadband polaron absorption. The clear distinction between absorption features from mobile polarons on the polymer (and anions) and undoped species suggest an integer-charge transfer, rather than partial charge transfer which would be shown as additional absorption features resulting from the formation of hybrid orbitals between PBTTT and $\mathrm{F}_{4}$-TCNQ. ${ }^{26}$ An efficient charge transfer was confirmed by current-voltage characteristics (Supplementary Section C), which shows a conductivity enhancement of six orders of magnitude (up to $250 \mathrm{~S} \mathrm{~cm}^{-1}$ at room temperature). The doping method was proven to be controllable in the full six orders of magnitude range by controlling the amount of $\mathrm{F}_{4}$-TCNQ evaporated on top of PBTTT and de-doping by annealing fully doped film (see Fig. S10 and S11, respectively). The extent of doping was confirmed by electron spin resonance (ESR) as discussed below, where we see a strong paramagnetic response with spin- $\frac{1}{2}$. These results all strongly suggests that $\mathrm{F}_{4}$-TCNQ molecules diffuse readily from the surface into the bulk and act as an efficient charge transfer dopant. Figure 1c shows a depth profile of the molecular ratio between $\mathrm{F}_{4}$-TCNQ and PBTTT for $15 \mathrm{~nm}$ of $\mathrm{F}_{4}$-TCNQ deposited on $40 \mathrm{~nm}$ of PBTTT film (see Supplementary Section B). The molecular ratio is estimated from X-ray photoemission spectroscopy (XPS) spectra for the Nitrogen $1 s$ peak (see the inset, and more details are shown in Supplementary Section B) as a function of sputtering depth. At the surface, the thermally evaporated $\mathrm{F}_{4}$-TCNQ molecules are present in excess, i.e. with a high molecular ratio. The XPS provides evidence for ready bulk diffusion of $\mathrm{F}_{4}$-TCNQ molecules all the way through the PBTTT film down to the substrate; in the bulk we observed a molecular ratio of $\sim 0.3$ corresponding to three PBTTT repeat units 
per one $\mathrm{F}_{4}$-TCNQ molecule. This molecular ratio is higher than the maximum molecular ratio used in solution-based co-deposition. ${ }^{28}$

Doping by mixing an organic donor into a conjugated polymer at such high doping levels is normally expected to cause some structural disorder. ${ }^{29}$ Surprisingly, we have found that the characteristic, lamellar microstructural order of PBTTT is preserved - even enhanced - upon doping as observed in grazing incidence wide angle X-ray scattering (GI-WAXS) images. Figure 1d shows GI-WAXS images for the doped PBTTT film. Several orders of (h00) diffraction peaks due to the lamellar spacing are observed in the out-of-plane scattering direction along $q_{\mathrm{z}}$. From X-ray diffraction (XRD) measurements (Figure 1e), the lamellar spacing of the pristine PBTTT film is calculated to be $22.9 \AA$ (from the peak at $q_{\mathrm{z}}=0.27 \AA^{-1}$; see Supplementary section A), which is consistent with a previous literature value for PBTTT-C $16 \cdot{ }^{30}$ The diffraction peaks for the doped PBTTT are found to shift to shorter scattering vector by $q_{\mathrm{z}}=0.20 \AA^{-1}$ (Fig. 1e), corresponding to a larger lamellar spacing of $24.7 \AA$ than the pristine PBTTT. On the other hand, the position of the in-plane diffraction peaks along $q_{\mathrm{xy}}$ (the inset in Fig. 1e) are insensitive to the doping. This suggests that the $\mathrm{F}_{4}$-TCNQ molecules reside primarily in the alkyl side-chain regions and as a result expand the lamellar spacing defined by the interdigitated side chains slightly, but importantly do not disturb the $\pi-\pi$ interaction between the polymer chains. We cannot exclude that some fraction of F4-TCNQ molecules is incorporated into the amorphous regions of the film, but we do not believe that this is a significant fraction as we cannot resolve any contribution from charge carriers on polymer chains with face-on orientation in the electron spin resonance ${ }^{31}$ (see Supplementary Section H). The high degree of order and intercalation of the dopant molecules in the side-chain regions is in contrast with the resulting structure from solution-based co-deposition ${ }^{28}$ which convincingly shows the dopant intercalation between the $\pi$ stacked conjugated chains from an unchanged lamellar spacing but a smaller $\pi$-stacking distance. Surprisingly, the interaction between the $\mathrm{F}_{4}$-TCNQ molecules and the side-chains even enhances the chain alignment in the polymer backbone plane, as confirmed from the X-ray rocking scan in Fig. 1f, where the full width at half maximum of a Gaussian fitted to the (100) diffraction is significantly smaller for the doped PBTTT than for the pristine one which indicates a greater degree of orientation of crystallites in the film. The overall consequence is that the dopants 
located in the insulating side chain regions inject carriers in the two-dimensional conjugated planes, but do not disrupt the critical $\pi$-stacking between the conjugated backbones. Our solid-state diffusion method results in a structure that may be regarded as an analogy to modulation-doped heterostructures in inorganic semiconductors. ${ }^{32}$ We were able to extend the doping method to several PBTTT polymers with different side-chain lengths $\left(\mathrm{C}_{10}, \mathrm{C}_{14}\right.$ and $\left.\mathrm{C}_{16}\right)$, Poly(3-hexylthiophene-2,5-diyl) (P3HT) as well as some high-mobility donor-acceptor copolymers with more complex chemical structures demonstrating some level of generality. We believe that the doping method could be applied to a wide range of conjugated polymers that owe their high mobilities to a well organised microstructure but for materials with deep ionisation potentials this might require the use of p-type dopants stronger than $\mathrm{F}_{4}$-TCNQ (see Supplementary Section E).

We now turn to a discussion of the charge transport properties of these highly conducting films. Probing spin susceptibility is a powerful way to distinguish the nature of electron localisation in organic conductors. ${ }^{31,33}$ Free electrons contribute to Pauli paramagnetic susceptibility, $\chi_{\text {Pauli }}$, which is expected to be temperature independent as long as the temperature is less than the Fermi temperature. Localised electrons, on the other hand, contribute to Curie susceptibility, $\chi_{\text {Curie }}{ }^{31}$ which tends to be larger rather than the Pauli susceptibility and exhibits a strong temperature dependence of the form $C / T$, where $C$ is the Curie constant, because all localised electron spins can respond to the external magnetic field. ESR enables measurement of the spin susceptibility from taking the double integral of the obtained derivative signal (Fig. 2a). The net susceptibility of the film can be written down as a linear sum of the two two contributions $\chi_{\text {tot }}=\chi_{\text {Pauli }}+\chi_{\text {curie }}$ and these components can be best extracted by using a so-called $\chi_{\text {tot }} T-T$ plot, i.e. $\chi_{\text {tot }} T=\chi_{\text {Pauli }} T+C$ (Figure $\left.2 \mathrm{~b}\right)$. The observed linear slope shows that there is a significant Pauli contribution from free-electron like states with relatively large density of states (DOS) at the Fermi energy $\rho\left(E_{\mathrm{F}}\right)=5.7 \pm 0.5 \times 10^{21} \mathrm{eV}^{-1} \mathrm{~cm}^{-3}$. The value of $\rho\left(E_{\mathrm{F}}\right)$ provides an insight to the low-dimensional nature of the transport in the $2 \mathrm{D}$ conjugated lamellae of PBTTT. The DOS for a two-dimensional degenerate Fermi gas is $\frac{m^{*}}{\pi \hbar^{2}}$, where $m^{*}$ is the effective mass of the charge carriers. Hence, each 2D conjugated-polymer backbone plane within the thickness of the polymer film is expected to contribute $\frac{m^{*}}{\pi \hbar^{2}}$ to the DOS which is consistent with the experimentally determined value if we assume a carrier mass of $2 m_{e}$, 
where $m_{e}$ is the free electron mass. In addition, $C$ measured from the finite intercept of $\chi_{\text {tot }} T-T$ plot is assigned to localised spins most likely from the $\mathrm{F}_{4}$-TCNQ anions, from which we estimate the temperature-independent anion spin concentration $n_{\text {spin }}=$ $3.3 \pm 0.2 \times 10^{20} \mathrm{~cm}^{-3}$ (see the Methods section).

The delocalised charge transport can be directly confirmed and elaborated further by discussing the Hall effect measured in a standard Hall-bar geometry shown in Fig. 2c (see Supplementary Section F). A clear Hall voltage signal was observed in a wide range of temperatures from $280 \mathrm{~K}$ to $20 \mathrm{~K}$ providing evidence for metallic behaviour of delocalised charge carriers. The symmetry and sign of the voltage correspond to hole charge carriers (Supplementary Section D). We simultaneously also measured the longitudinal conductivity $\sigma$ which does not exhibit an ideal metallic behaviour but increases with increasing temperature according to $\sigma(T)=\sigma_{0} \exp \left(\frac{T_{0}}{T}\right)^{-\frac{1}{d}}$, where $T_{0}$ is a characteristic temperature. The experimental data can be fitted well with $d=2.34$ over the whole temperature range and agrees locally with $d=2$ at low temperatures that is predicted by the Efros-Shklovskii variable-range-hopping (VRH) model ${ }^{34}$ and other hopping models. We do not want to speculate here about the mechanism (more details in Supplementary Section I), but would only like to note that the positive temperature coefficient of conductivity does not contradict the existence of metallic states, because the Hall voltage can be generated by summing over contributions from metallic grains where Hall voltages are produced, even if there are grain boundaries in between that prevent observation of a fully metallic longitudinal conductivity. ${ }^{35}$ Hopping transport between localised states cannot explain the observed Hall effect, as it would result in only a small contribution to Hall voltage due to the modulation of transition probabilities in inter-site hopping by magnetic field $^{36}$ and would be expected to result as well in an opposite sign of the Hall coefficient ("sign anomaly") 37,38 in contrast to the observed behaviour. We find the Hall mobility $\mu_{\mathrm{H}}=\sigma R_{\mathrm{H}}$ to be near temperature-independent around $300 \mathrm{~K}$, but to decrease as well with decreasing temperature at lower temperatures (see Fig. 2i) The ratio of the room temperature conductivity to that at $20 \mathrm{~K}, \sigma(300 \mathrm{~K}) / \sigma(20 \mathrm{~K})=7$ is however larger than that of the Hall mobility, $\mu_{\mathrm{H}}(300 \mathrm{~K}) / \mu_{\mathrm{H}}(20 \mathrm{~K})=3$, reflecting that the conductivity is more strongly affected by grain boundaries than the Hall mobility. The room temperature Hall mobility of $1.8 \mathrm{~cm}^{2} \mathrm{~V}^{-1} \mathrm{~s}^{-1}$ is one of the highest values reported for a conducting 
polymer $23,24,39$ and a factor of two higher than the reported FET mobility. ${ }^{40}$ We can use the ESR results to estimate the concentration of mobile charge carriers (shown as the black line in Fig. 2h) that should match the inverse Hall coefficient if the Hall effect had an ideal signature. At room temperature the inverse Hall coefficient is larger than the total charge density estimated from $C$, indicating a partially decoherent charge transport. ${ }^{16}$ However, the deviation becomes smaller as the temperature decreases and the system exhibits an ideal Hall effect at low temperatures. This suggests that at low temperatures the transport in the metallic grain becomes increasingly coherent. Our analysis relies on the assumption that each $\mathrm{F}_{4}$-TCNQ anion manifesting itself as a localised spin in ESR produces one mobile charge carrier on the polymer. Such near unity dissociation probability for cation-anion pairs into mobile holes on the polymer is not an unreasonable assumption at high carrier concentrations as shown recently. ${ }^{41}$ Our estimate of charge carrier concentration on the polymer is also consistent with independent measurements of the film composition and the degree of charge transfer (Supplementary Section B).

This observation of coherent charge transport at low temperature is fully consistent with longitudinal magnetoconductance measurements. Upon applying a perpendicular magnetic field, the conductivity of the film increased (Fig. 3a), more strongly so at low temperatures. The positive magnetoconductance (MC) can be interpreted in terms of the weak-localisation (WL) effect arising from coherent backscattering due to a constructive interference between electron waves traversing along closed paths in opposite senses with equal probabilities. ${ }^{42}$ The symmetry is broken by the magnetic field flux enclosed by the paths and this reduces backscattering and enhances conductivity. WL phenomenon has been reported as a positive $\mathrm{MC}$ in $\operatorname{poly}(p$-phenylenevinylene $),{ }^{43}$ doped polyacetylene, ${ }^{44,45}$ polyaniline ${ }^{46}$ and electrolyte gated $\mathrm{PBTTT}^{47}$ but the effect could only be observed at very low temperatures (sub-5 K). The positive MC observed here over a wide temperature range is in agreement with a well-established WL framework, the Hikami-Larkin-Nagaoka (HLN) model. ${ }^{48}$ Assuming weak spin-orbit coupling (see Methods), the magnetoconductance is parabolic with the applied field, given as $\Delta G(B)=\frac{G_{0}}{24}\left(\frac{B}{B_{\varphi}}\right)^{2}$, where $G_{0}=\frac{e^{2}}{2 \pi^{2} \hbar}$. $B_{\varphi}$ is the characteristic field for which the matrix element for backscattering loses its phase and is the only fitting parameter that can reproduce both the magnitude and curvature of the positive MC (shown in Fig. 3b). $B_{\varphi}$ can be directly related to the inelastic scattering time (Fig. 3c), 
$\tau_{\varphi}$, by $B_{\varphi}=\frac{\hbar}{4 e D \tau_{\varphi}}$, where $D$ is the diffusion constant of charge motion. Assuming the Einstein's relation holds, $\tau_{\varphi}$ can be related to inelastic scattering length (Fig. $\left.3 \mathrm{~d}\right), \lambda_{\varphi}$, via $\lambda_{\varphi}=\sqrt{D \tau_{\varphi}}$, by estimating $D=\frac{\mu_{\mathrm{H}} k_{\mathrm{B}} T}{e}$. Inelastic scattering destroys the phase coherence of delocalised charge wavepackets and this happens at characteristic time- and length-scales of $\tau_{\varphi}$ and $\lambda_{\varphi}$, respectively. The destructive interference becomes apparent when $\lambda_{\varphi}$ is comparable to the Landau orbit size, ${ }^{42} L=\sqrt{\hbar / e B}$, which is $9 \mathrm{~nm}$ at $8 \mathrm{~T}$ and of the same order as $\lambda_{\varphi}$, indeed. Both $\tau_{\varphi}$ and $\lambda_{\varphi}$ increase at low temperatures, in agreement with the Hall measurements which indicate a greater degree of charge coherence at low temperatures. In addition, the temperature dependence of $\tau_{\varphi}$ hints towards electron-electron scattering as a dominant scattering mechanism from the $T^{-2}$ fit. ${ }^{48}$ In this study, $D$ can be accurately determined to extract $\tau_{\varphi}$ and $\lambda_{\varphi}$ over a wide temperature range and such comprehensive analysis from concurrent measurement of WL and Hall effect have not been reported to probe the coherence of charge transport in conducting polymer to the best of our knowledge. The above formalism of weak-localisation is formulated for a two-dimensional system, and therefore magnetoconductance supports the notion of the quasi-2D nature of charge delocalisation in our system, which we expect from the anisotropic packing structure of the polymer, with the alkyl side chains preventing charge delocalisation along the out-of-plane direction.

Our interpretation of the magnetoconductance is confirmed by the angular dependence. When varying the angle that the magnetic field makes with the substrate plane, the measured magnetoconductance was found to be composed of two components, as seen from the fit at $35 \mathrm{~K}$ in Fig. 3e, and can be expressed as $\Delta G=\Delta G_{\mathrm{WL}}+\Delta G_{\mathrm{ee}}$, where $\Delta G_{\mathrm{WL}}$ is an angular dependent, 2D weak-localisation component and $\Delta G_{\mathrm{ee}}$ a component isotropic with the field direction (see Supplementary Section G). We expect the weak-localisation effect to depend on the magnetic flux, and therefore $\Delta G_{\mathrm{WL}} \propto\left(\frac{B \cos (\theta)}{B_{\varphi}}\right)^{2}$. The angular dependence of magnetoconductance at $8 \mathrm{~T}(\mathrm{MC}(B=8 \mathrm{~T}))$ is well reproduced with a combination of $\cos ^{2}(\theta)$ and a $B$-independent MC offset.

It is interesting to contrast the coherent charge transport in PBTTT/ $\mathrm{F}_{4}-\mathrm{TCNQ}$ with those of other, state-of-the-art conducting polymers with more disordered microstructure. Magnetotransport measurements were also performed for poly(3,4-ethylenedioxythiophene): 
polystyrene sulfonate (PEDOT:PSS, Fig. 4a) with the same Hall-bar geometry. PEDOT:PSS exhibits a disordered, amorphous microstructure in 2D GI-WAXS (Fig. 4b). The ESR measurement of a PEDOT:PSS film shows a negligible contribution from Pauli susceptibility; no slope in $\chi_{\text {tot }} T-T$ can be observed but follows Curie law, i.e. $\chi^{-1} \propto T$ (see Fig. $4 \mathrm{~d}$ ). This is further reflected in the Hall effect measurement which was challenging due to a small signal-to-noise ratio as seen from the Fig. 4e. This is expected to be the result of a decoherent charge transport which manifests itself as a small Hall mobility of $0.2 \mathrm{~cm}^{2} \mathrm{~V}^{-1} \mathrm{~s}^{-1}$, an order of magnitude lower than that of PBTTT $/ \mathrm{F}_{4}$-TCNQ, despite having six times higher conductivity (details are summarised in Fig. 4g-i). The magnetoconductance is negative, representing a dominant contribution of magnetic-field-induced localisation of wavefunction, and the weak-localisation-signature seen in PBTTT/F $/ \mathrm{F}_{4}$ TCNQ is absent (Fig. 4f). In addition, a good fit of the temperature dependence of conductivity with three dimensional VRH model in Fig. $4 \mathrm{~g}$ is consistent with a predominantly hopping transport in PEDOT:PSS. The discrepancy between the estimates of charge density from ESR and Hall measurements is significant over the whole temperature range. The Hall measurement overestimates the charge density by a factor of 500 (Fig. 4.h), clearly demonstrating a non-ideal Hall effect, with a coherence factor much smaller than $1 .^{16}$

The Hall mobility of PBTTT/ $/ F_{4}-\mathrm{TCNQ}$ is significantly higher than what has been measured in other conducting polymers. Except for specially prepared polyaniline, ${ }^{10}$ which shows a truly metallic charge transport, the room temperature Hall mobility of $\mathrm{PBTTT} / \mathrm{F}_{4}-\mathrm{TCNQ}$ is unprecedented. For example, polyacetylene doped with $\mathrm{AsF}_{5}$ shows two orders of magnitude lower Hall mobility ${ }^{23}$ than PBTTT/F F TCNQ despite having six times higher conductivity. The striking difference in the Hall mobility is likely to arise from the different degree of microstructural order present in highly doped polymer films. Normally, charge-transfer leaves behind counter-ions which induce structural and energetic disorder when incorporated into the conjugated domains and may also act as scattering centres. In PBTTT $/ \mathrm{F}_{4}$-TCNQ the dopant molecules become incorporated into the side-chain regions and do not disturb the semi-crystalline lamellar $\pi$-stacking of the polymer backbones. This minimises the disorder and allows charge to delocalise sufficiently to exhibit the signatures of coherent, metallic transport discussed above. In order to interpret the relatively low conductivity value (up to $250 \mathrm{~S} \mathrm{~cm}^{-1}$ ) compared to conventional highly doped polymers, it is important to consider the $2 \mathrm{D}$ nature of charge transport. 
In $\mathrm{PBTTT} / \mathrm{F}_{4}$-TCNQ the charge concentration is orders of magnitude smaller than in polyacetylene $^{45}$ and polyaniline ${ }^{10}$ which exhibit more compact packing of polymer chains due to the absence of the alkyl sidechains which are needed to impart solution processibility. It is interesting to correct the conductivity for the volume occupied by the insulating side chains (taking the thiophene ring size of $3.60 \AA$ ) and estimate the "effective conductivity" of the conjugated lamellae which is up to six times higher than the average bulk conductivity, i.e. on the order of $1000 \mathrm{~S} \mathrm{~cm}^{-1}$. This is comparable to that of polyaniline or polyacetylene. Although this "effective conductivity" is not of direct practical relevance, it nevertheless demonstrates further the excellent charge transport properties of our system.

Our results demonstrate that it is possible to retain a high level of microstructural order in a conducting polymer, even at high doping levels. Our method of incorporating the dopant molecules by solid-state diffusion, which is akin to diffusion-based doping processes used in inorganic semiconductors, retains, and even improves, the highly semicrystalline microstructure of the undoped conjugated polymer and allows us to selectively incorporate the dopants into electrically inactive regions of the film occupied by the insulating, flexible side chains. This enables observation of novel transport phenomena in conjugated polymers that rely on coherence of the wavefunction on at least several nm's. Our approach which may be seen to be analogous to modulation doping in inorganic semiconductor heterostructures opens new opportunities for studying in a more controlled way the structure-property relationships of conducting polymers at high carrier concentrations and the rich, coherent and correlated electron transport physics that is expected in this regime if disorder effects can be overcome. Our method is applicable to a wide range of conducting polymer systems and will enable the improvement of the transport properties of conducting polymers used in a wide range of applications, such as electrodes in optoelectronic devices or as the active conducting layer in thermoelectric converters.

1 Yu, G., Gao, J., Hummelen, J., Wudl, F. \& Heeger, A. Polymer photovoltaic cells: Enhanced efficiencies via a network of internal donor-acceptor heterojunctions. Science 270, 1789-1791 
(1995).

2 Meijer, E. et al. Solution-processed ambipolar organic field-effect transistors and inverters. Nature materials 2, 678-682 (2003).

3 Alves, H., Molinari, A. S., Xie, H. \& Morpurgo, A. F. Metallic conduction at organic chargetransfer interfaces. Nature materials 7, 574-580 (2008).

${ }^{4}$ Gutiérrez Lezama, I. et al. Single-crystal organic charge-transfer interfaces probed using Schottky-gated heterostructures. Nature materials 11, 788-94 (2012).

${ }^{5}$ Chiang, C. K. et al. Electrical conductivity in doped polyacetylene. Physical Review Letters 39, 1098 (1977).

${ }^{6}$ Bryce, M. R. Recent progress on conducting organic charge-transfer salts. Chemical Society Reviews 20, 355 (1991).

7 Friend, R. Conductive Polymers II: From Science to Applications. No. v. 2 in RAPRA Technology Limited Shawbury: RAPRA review reports (Rapra Technology, 1993).

8 Inzelt, G. Applications of conducting polymers. In Conducting Polymers, 245-293 (Springer, 2012).

9 Kiebooms, R., Aleshin, a., Hutchison, K. \& Wudl, F. Thermal and Electromagnetic Behavior of Doped Poly(3,4-ethylenedioxythiophene) Films. The Journal of Physical Chemistry B 101, 11037-11039 (1997).

10 Lee, K. et al. Metallic transport in polyaniline. Nature 441, 65-68 (2006).

11 Bubnova, O. et al. Semi-metallic polymers. Nature materials 13, 190-4 (2014).

12 Chang, Y., Lee, K., Kiebooms, R., Aleshin, A. \& Heeger, A. J. Reflectance of conducting poly(3,4-ethylenedioxythiophene). Synthetic Metals 105, 203-206 (1999).

13 Ikehata, S. et al. Solitons in Polyacetylene: Magnetic Susceptibility. Physical Review Letters 45, 1123-1126 (1980).

14 Podzorov, V., Menard, E., Rogers, J. \& Gershenson, M. Hall Effect in the Accumulation Layers on the Surface of Organic Semiconductors. Physical Review Letters 95, 226601 (2005).

15 Chang, J.-F. et al. Hall-Effect Measurements Probing the Degree of Charge-Carrier Delocalization in Solution-Processed Crystalline Molecular Semiconductors. Physical Review Letters 107, 066601 (2011).

16 Uemura, T. et al. Temperature dependence of the Hall effect in pentacene field-effect transistors: Possibility of charge decoherence induced by molecular fluctuations. Physical Review B $\mathbf{8 5}$, 
$035313(2012)$.

17 Wang, S., Ha, M., Manno, M., Daniel Frisbie, C. \& Leighton, C. Hopping transport and the Hall effect near the insulator-metal transition in electrochemically gated poly(3-hexylthiophene) transistors. Nature communications 3, 1210 (2012).

18 Lee, B. et al. Trap healing and ultralow-noise Hall effect at the surface of organic semiconductors. Nature materials 12, 1125-9 (2013).

19 Yamashita, Y. et al. Transition Between Band and Hopping Transport in Polymer Field-Effect Transistors. Advanced Materials 26, 8169-8173 (2014).

20 Senanayak, S. P., Ashar, a. Z., Kanimozhi, C., Patil, S. \& Narayan, K. S. Room-temperature bandlike transport and Hall effect in a high-mobility ambipolar polymer. Physical Review B 91, $115302(2015)$.

21 Friedman, L. Hall Conductivity of Amorphous Semiconductors in the Random Phase Model. Journal of Non-Crystalline Solids 6, 329-341 (1971).

22 Klein, R. S. Investigation of the Hall effect in impurity-hopping conduction. Physical Review B 31, 2014-2021 (1985).

23 Seeger, K., Gill, W., Clarke, T. \& Street, G. Conductivity and hall effect measurements in doped polyacetylene. Solid State Communications 28, 873-878 (1978).

24 Shacklette, L. W., Chance, R. R., Ivory, D. M., Miller, G. G. \& Baughman, R. H. Electrical and optical properties of highly conducting charge-transfer complexes of poly ( p-phenylene). Synthetic Metals 1, 307-320 (1980).

25 MacDiarmid, A. G. \& Chiang, J. C. Polyanline: Protonic acid doping of the emeraldine form to the metallic regime. Synthetic Metals 13, 193-205 (1986).

26 Aziz, E. F. et al. Localized Charge Transfer in a Molecularly Doped Conducting Polymer. Advanced Materials 19, 3257-3260 (2007).

27 Zhang, Y., de Boer, B. \& Blom, P. W. M. Controllable Molecular Doping and Charge Transport in Solution-Processed Polymer Semiconducting Layers. Advanced Functional Materials 19, 1901-1905 (2009).

28 Cochran, J. E. et al. Molecular interactions and ordering in electrically doped polymers: Blends of pbttt and f4tcnq. Macromolecules 47, 6836-6846 (2014).

29 Heeger, a. J., Kivelson, S., Schrieffer, J. R. \& Su, W. P. Solitons in conducting polymers. Reviews of Modern Physics 60, 781-850 (1988). 
30 Kline, R. J. et al. Critical role of side-chain attachment density on the order and device performance of polythiophenes. Macromolecules 40, 7960-7965 (2007).

31 Tanaka, H., Hirate, M., Watanabe, S. \& Kuroda, S.-i. Microscopic signature of metallic state in semicrystalline conjugated polymers doped with fluoroalkylsilane molecules. Advanced Materials 26, 2376-2383 (2014).

32 Dingle, R., Störmer, H. L., Gossard, a. C. \& Wiegmann, W. Electron mobilities in modulationdoped semiconductor heterojunction superlattices. Applied Physics Letters 33, 665-667 (1978).

33 Mizoguchi, K. et al. Pauli and curie susceptibilities of polythiophene. Synthetic Metals 18, 195-198 (1987).

34 Efros, A. L. \& Shklovskii, B. I. Coulomb gap and low temperature conductivity of disordered systems (2001).

35 Orton, J. \& Powell, M. The Hall effect in polycrystalline and powdered semiconductors. Reports on Progress in Physics 1263 (1980).

36 Holstein, T. Hall effect in impurity conduction. Physical Review 124, 1329-1347 (1961).

37 Le Comber, P. G., Jones, D. I. \& Spear, W. E. Hall effect and impurity conduction in substitutionally doped amorphous silicon (1977).

38 Gilani, T. H., Masui, T., Yu. Logvenov, G. \& Ishiguro, T. Low-temperature Hall effect and thermoelectric power in metallic PF6-doped polypyrrole. Synthetic Metals 78, 327-331 (1996).

39 Long, V. C., Washburn, S., Chen, X. L. \& Jenekhe, S. a. Hall-effect study of an ion-bombarded polymer. Journal of Applied Physics 80, 4202-4204 (1996).

$40 \mathrm{McCulloch}$, I. et al. Liquid-crystalline semiconducting polymers with high charge-carrier mobility. Nature Materials 5, 328-333 (2006).

41 Mityashin, A. et al. Unraveling the mechanism of molecular doping in organic semiconductors. Advanced Materials 24, 1535-1539 (2012).

42 Lee, P. A. \& Ramakrishnan, T. Disordered electronic systems. Reviews of Modern Physics 57, 287-337 (1985).

43 Ahlskog, M., Reghu, M., Heeger, a., Noguchi, T. \& Ohnishi, T. Electronic transport in the metallic state of oriented poly(p-phenylenevinylene). Physical Review B 53, 15529-15537 (1996).

44 Väkiparta, K. et al. Anisotropy of magnetoconductivity in oriented poly(acetylene) doped with iodine (1993). 

toresistance features in heavily doped polyacetylene. Physical Review B 64, 1-5 (2001).

46 Mukherjee, A. K. \& Menon, R. Magnetotransport in doped polyaniline. Journal of Physics: Condensed Matter 17, 1947-1960 (2005).

47 Zanettini, S. et al. Magnetoconductance anisotropy of a polymer thin film at the onset of metallicity. Applied Physics Letters 106, 063303 (2015).

48 Hikami, S., Larkin, A. \& Nagaoka, Y. Spin-orbit interaction and magnetoresistance in the two dimensional random system. Progress of Theoretical Physics 63, 707-710 (1980).

\section{Acknowledgements}

The research leading to these results has received funding from the European Research Council under the European Union's Seventh Framework Programme (FP7/2007-2013) / ERC grant agreement n 610115. K.K. thanks the Samsung Scholarship Foundation for financial support. S.W. thanks H. Matsui and J. Takeya of University of Tokyo for stimulating discussions, and is supported by Research Fellowships of Japan Society for the Promotion of Science for Young Scientists, and JST PRESTO. K.B. acknowledges funding by the German Research Foundation (BR 4869/1-1). S. K. and H. T. acknowledge funding from the Japan Society for the Promotion of Science (No. 25287073). The authors thank D. Venkateshvaran and A. Sadhanala of the University of Cambridge for help with the measurements. Part of this work is based upon research conducted at the Cornell High Energy Synchrotron Source (CHESS). CHESS is supported by the NSF \& NIH/NIGMS via NSF award DMR-1332208. We thank D.-M. Smilgies, X. Sheng and J. Dolan for their help during the D1 experiment at CHESS. We thank A. Hexemer, R. Pandolfi and C. Zhu for supporting the data evaluation, who are supported by the U.S. Department of Energy under Contract No. DEAC02-05CH1123, the ECA award program and the LBNL LDRD ?TReXS?. The authors thank Professor Klaus Müllen of Max Planck Institue for Polymer Research for providing CDT-BTZ.

\section{Author contributions}

K.K. and S.W. conceived, designed, performed the experiments, fabricated all the samples, analysed the data, and wrote the manuscript with significant input from H.S. All X-ray measurements were done by K.B. and A.S.. A. B. performed XPS measurements. I. N. measured temperature dependent UV-Vis optical abosrption spectrum of PBTTT/F 4 -TCNQ and M. 
N. performed FTIR measurements (Suppelementary information). ESR measurements were performed by H.T. and S.K. (for PBTTT $/ \mathrm{F}_{4}-\mathrm{TCNQ}$ ), and by D.M. and K.M (for PEDOT/PSS). Z.F. and M.H. synthesised and purified the PBTTT. S.W. and H.S. supervised this work. All authors discussed the results and reviewed the manuscript.

\section{Additional information}

The authors declare no competing financial interests. 


\section{Methods}

\section{Device fabrication}

The devices were made on a $0.5 \mathrm{~mm}$-thick sapphire substrate cleaned by a sonication cleaning processes in deionized water, acetone, and isopropanol. Sapphire was used to minimize current-induced heating effects in the electrical measurements. After cleaning with an oxygen plasma treatment, electrodes were lithographically formed via a standard double-layer lift off process. The electrodes consisting of chromium (Cr, $1.5 \mathrm{~nm}$, for adhesion) and gold (Au, $20 \mathrm{~nm}$ ) were thermally evaporated at the base pressure of $1 \times 10^{-6}$ mbar. Channel length $(L)$ and width $(W)$ were $240 \mu \mathrm{m}$ and $80 \mu \mathrm{m}$ with four probes mounted in between source and drain electrodes (see Fig. 2c). On top of the electrodes, PBTTT film was spin-coated from a $10 \mathrm{mg} \mathrm{ml}^{-1}$ 1,2-dichlorobenzene solution in a nitrogen glovebox to form a $40 \mathrm{~nm}$ thick film, which was annealed at $180{ }^{\circ} \mathrm{C}$ for 20 mins, then slowly cooled down to room temperature. The $\mathrm{F}_{4}$-TCNQ was thermally evaporated directly on the top of the PBTTT layer from a powder purchased from Sigma-Aldrich at a base pressure of $2 \times 10^{-7} \mathrm{mbar}$ with a rate of $1-2 \AA \mathrm{s}^{-1}$ upto a nonminal thickness of $25 \mathrm{~nm}$. For such thick coverage not all $\mathrm{F}_{4}$-TCNQ diffused into the polymer film underneath, but a layer of neutral $\mathrm{F}_{4}$-TCNQ molecules remained on the surface. The diffusion readily occured during the deposition and room temperature storage. The resulting doped film was patterned into a Hall bar by photolithography combined with etching by oxygen plasma ashing. More details are given in Supplementary information Section A and C.

\section{Analysis of ESR spectra}

The Pauli susceptibility is expressed as $\chi_{\text {Pauli }}=\mu_{\mathrm{B}}^{2} \rho\left(E_{\mathrm{F}}\right)$, where $\mu_{\mathrm{B}}$ and $\rho\left(E_{\mathrm{F}}\right)$ are the Bohr magneton and the density of states at the Fermi energy. At a low magnetic field or high temperature limit, the Curie susceptibility is expressed $\mathrm{as}^{31} ; \chi_{\text {Curie }}=\frac{\mu_{\mathrm{B}}^{2}}{3 k_{\mathrm{B}} T} N_{\text {spin }} g^{2} S(S+1)$, where $k_{\mathrm{B}}, N_{\text {spin }}, g$ and $S$ are the Boltzmann constant, total spin concentration, $g$-factor, and the spin quantum number $(S=1 / 2)$. Moreover, it should be possible to distinguish the Pauli and Curie susceptibilities from their respective temperature dependence, if the net susceptibility is a linear sum of two components, i.e., $\chi_{\text {tot }}=\chi_{\text {Pauli }}+\chi_{\text {Curie }}$. From so-called $\chi_{\text {tot }} T-T$ plot, $\chi_{\text {tot }} T=\chi_{\text {Pauli }} T+C$, where $C$ is the Curie constant, both $\chi_{\text {Pauli }}$ and $C$ are determined as the gradient and intercept of the plot (Fig. 2b). We considered not only the spin states on a conjugated polymer, but also the contribution of spins from a counter anion on dopant because $g$-values measured for PBTTT and $\mathrm{F}_{4}$-TCNQ are accidentally 
equal (Supplementary Section H). It has been established that in a conjugated polymer positive charge carriers exist potentially in the form of i) localised polarons $(+e, s=1 / 2)$, ii) localised bipolarons $(+2 e, s=0)$, and iii) delocalised quasi-free hole states $(+e, s=1 / 2) .{ }^{29}$ As described above, ESR measures Curie susceptibility for i), and Pauli for iii), and ESR is completely silent for bipolaron state ii). Given the fact that the spins on anions induced by charge transfer should strongly localise at $\mathrm{F}_{4}$-TCNQ molecules and bipolarons are unlikely to exist in a small molecule, the anions in $\mathrm{F}_{4}$-TCNQ contribute purely to the spin- $1 / 2$ Curie susceptibility. Therefore, from the intercept of the $\chi_{\text {tot }} T-T$ plot, $2.08 \times 10^{-4}$ emu $\mathrm{K} \mathrm{cm}^{-3}$, we estimate the temperature-independent $n_{\text {spin }}$ to be $3.3 \pm 0.2 \times 10^{20}$ $\mathrm{cm}^{-3}$ with experimentally measured $g=2.00290$. We also conclude from the temperature independence of the UV-vis absorption measurement that the degree of charge transfer is indeed temperature independent (Supplementary Section F).

\section{Analysis of magnetoconductance}

The full HLN equation with weak spin-orbit field is,

$$
\Delta G(B)=-\frac{e^{2}}{2 \pi^{2} \hbar}\left[\Psi\left(\frac{1}{2}+\frac{B_{1}}{B}\right)-\Psi\left(\frac{1}{2}+\frac{B_{2}}{B}\right)+\frac{1}{2} \Psi\left(\frac{1}{2}+\frac{B_{3}}{B}\right)-\frac{1}{2} \Psi\left(\frac{1}{2}+\frac{B_{4}}{B}\right)\right]
$$

where the prefactor, $\frac{e^{2}}{2 \pi^{2} \hbar}$, is the quantised conductivity and $\Psi$ the digamma function. The parameters $B_{n}$ are linear combination of characteristic fields, $B_{i}$ :

$$
\begin{aligned}
B_{1} & =B_{e}+B_{\mathrm{so}}+B_{\mathrm{m}} \\
B_{2} & =\frac{4}{3} B_{\mathrm{so}}+\frac{2}{3} B_{\mathrm{m}}+B_{\varphi} \\
B_{3} & =2 B_{\mathrm{m}}+B_{\varphi} \\
B_{4} & =\frac{2}{3} B_{\mathrm{so}}+\frac{4}{3} B_{\mathrm{m}}+B_{\varphi}
\end{aligned}
$$

where $B_{i}$ and scattering times, $\tau_{i}$, are related by

$$
B_{i}=\frac{\hbar}{4 e D \tau_{i}}
$$


The index $i$ represents different scattering processes: elastic scattering, $e$, inelastic scattering, $\varphi$, spin-orbit scattering, so, and magnetic scattering, $m$. In the absence of spin-orbit scattering and magnetic impurities, both $B_{\text {so }}$ and $B_{\mathrm{m}}$ are negligible. In addition, if $B_{\mathrm{e}}>>B_{\varphi}$ the equation 1 simplifies to $\Delta G_{\mathrm{WL}}(B)=\frac{1}{24} \frac{e^{2}}{2 \pi^{2} \hbar}\left(\frac{B}{B_{\varphi}}\right)^{2}$. This is a reasonable assumption provided that intramolecular vibrations occur at much faster time-scales than intermolecular vibrations. Note that we did not take into account weak anti-localisation due to the presence of a spin-orbit field. A finite contribution of spin-orbit field initially breaks weak localisation even without the external magnetic field. 

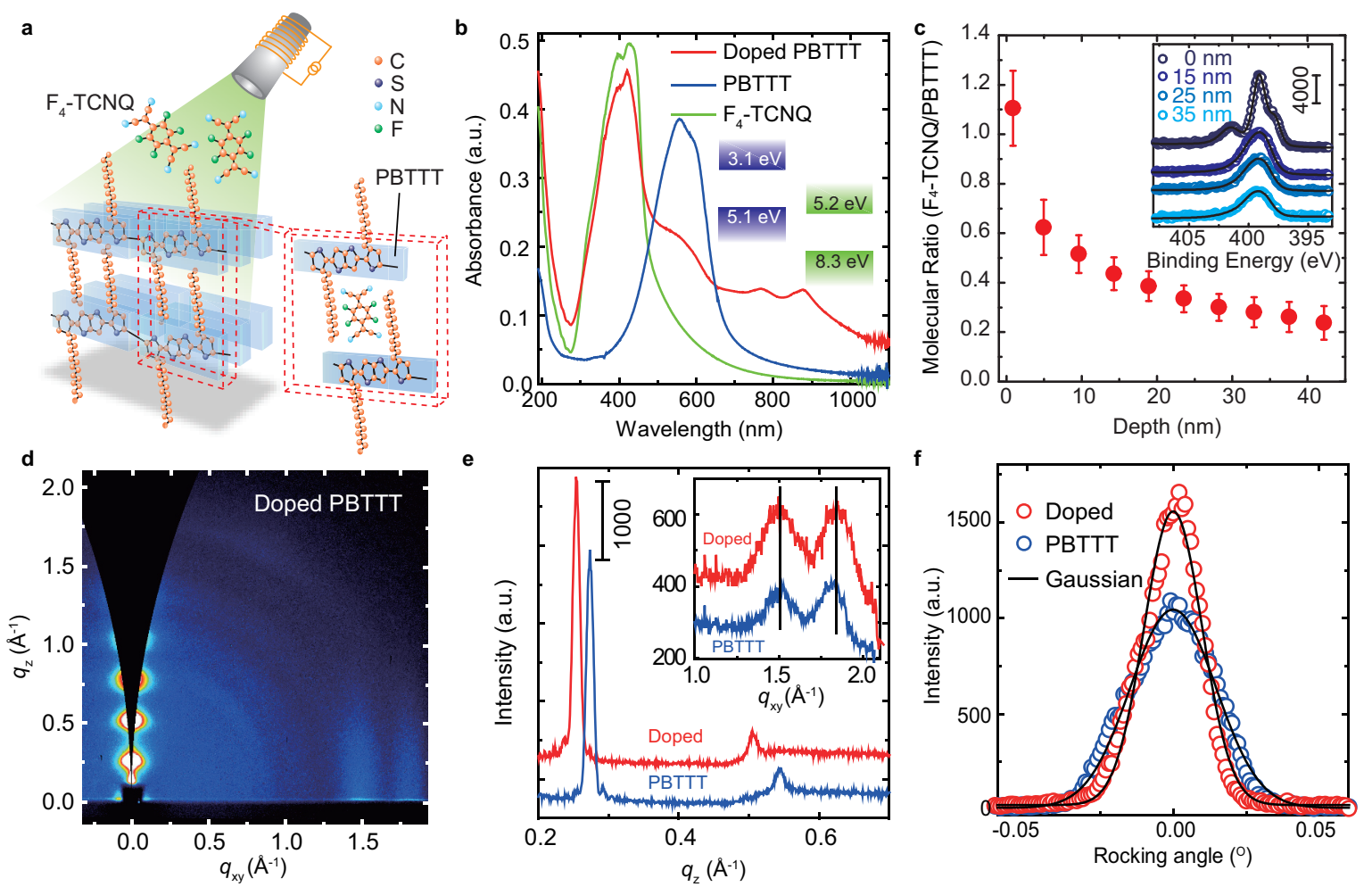

Figure 1: Highly ordered microstructure of doped PBTTT/F F $_{4}$ TCNQ. a, A schematic diagram of the doping method showing the evaporation procedure of $\mathrm{F}_{4}$-TCNQ and resulting edgeon stacking of PBTTT with $\mathrm{F}_{4}$-TCNQ molecules diffusing into inter-digitated sidechain region. The molecular structures of PBTTT and $\mathrm{F}_{4}$-TCNQ are presented with circles of different colours representing different atoms as described in the legend. b, Optical absorption spectra for doped PBTTT, pristine PBTTT, and pristine $\mathrm{F}_{4}$-TCNQ. Schematic energy diagram of PBTTT and $\mathrm{F}_{4^{-}}$ TCNQ is shown in the inset. ${ }^{28} \mathbf{c}$, XPS depth profile of the molecular ratio of $\mathrm{F}_{4}$-TCNQ to PBTTT. The error bars represent uncertainty in fitting from XPS measurements (see Supplementary Section B). The inset shows the detailed scan for the nitrogen $1 s$ peak at different depths of the film. d, 2D grazing incidence wide-angle X-ray scattering (GIWAXS) image for doped PBTTT. e, Specular scans from X-ray diffraction (XRD) measurements for out-of-plane scattering profile along $q_{\mathrm{z}}$ for doped and pristine PBTTT. The inset shows in-plane diffraction spectra along $q_{\mathrm{xy}}$ from GIWAXS. $q_{\mathrm{xy}}$ and $q_{\mathrm{z}}$ are the in-plane and the normal components of the scattering vector. f, Rocking scans for the (100) diffraction peaks. Black curves denote Gaussian fits, where the full width at hall maximum values are measured to be $0.023^{\circ}$ for doped PBTTT, and $0.032^{\circ}$ for pristine PBTTT. All the measurements were performed at room temperature. 

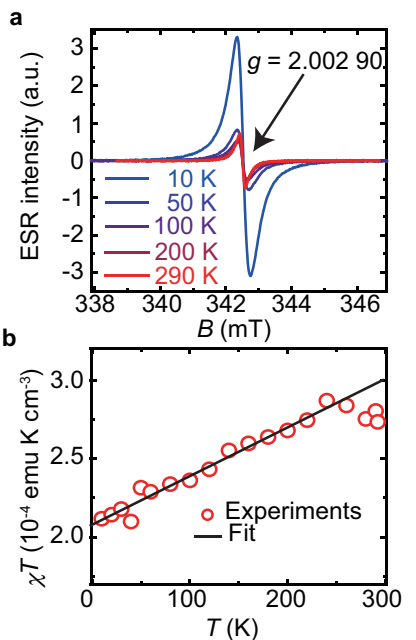
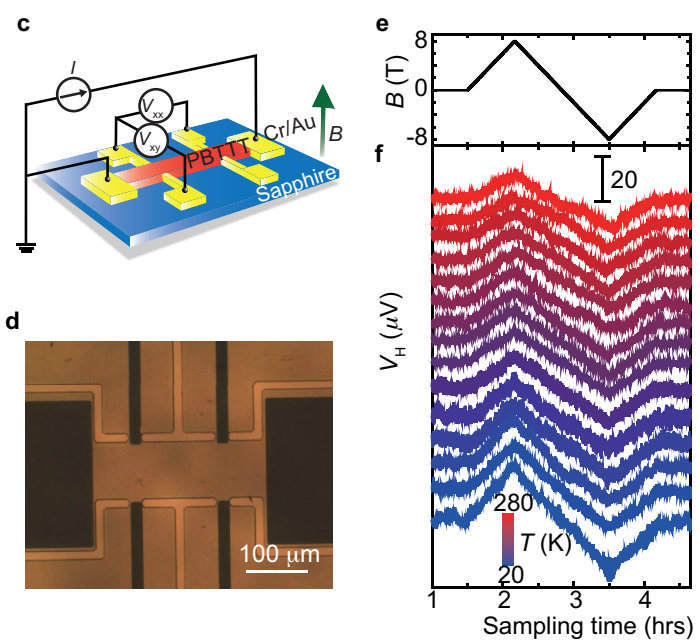

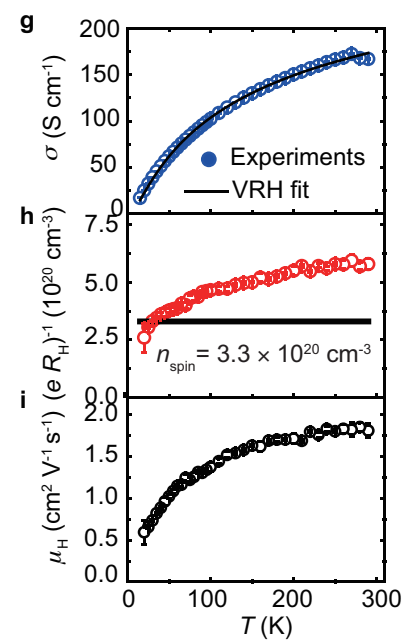

Figure 2: Delocalised charge transport in doped PBTTT/F F $_{4}$ TCNQ. a, ESR spectra measured for doped PBTTT at various temperatures; the external magnetic field is applied perpendicular to the film plane. $\mathbf{b}, \chi T-T$ plot for the spin susceptibility $(\chi)$ obtained from a double integration of the ESR spectra. c, Schematic illustration and $\mathbf{d}$, optical micrograph of the Hall bar. The longitudinal voltage $\left(V_{\mathrm{xx}}\right)$ and transverse voltage $\left(V_{\mathrm{xy}}\right)$ were recorded simultaneously with applying a constant, dc current $(I)$. $B$ was ramped up to $8 \mathrm{~T}$ and down to $-8 \mathrm{~T}$ during the measurements as shown in e. f, $V_{\mathrm{H}}$ measured at various temperatures $(T)$ from $20 \mathrm{~K}$ to $280 \mathrm{~K}$; the scale bar represents $20 \mu \mathrm{V} . \mathbf{g}, T$ dependence of the longitudinal conductivity $(\sigma)$. The contact resistance was corrected for by employing four-point-probe measurements. $\mathbf{h}, T$ dependence of the inverse Hall coefficient $R_{\mathrm{H}}^{-1}$, together with the spin concentration $\left(n_{\text {spin }}\right)$ estimated from the Curie component of ESR signals (black line). The thickness of the line represents the error from fitting with Curie law. i, $T$ dependence of the Hall mobility $\mu_{\mathrm{H}}$ estimated from $\mu_{\mathrm{H}}=R_{\mathrm{H}} \sigma$. 

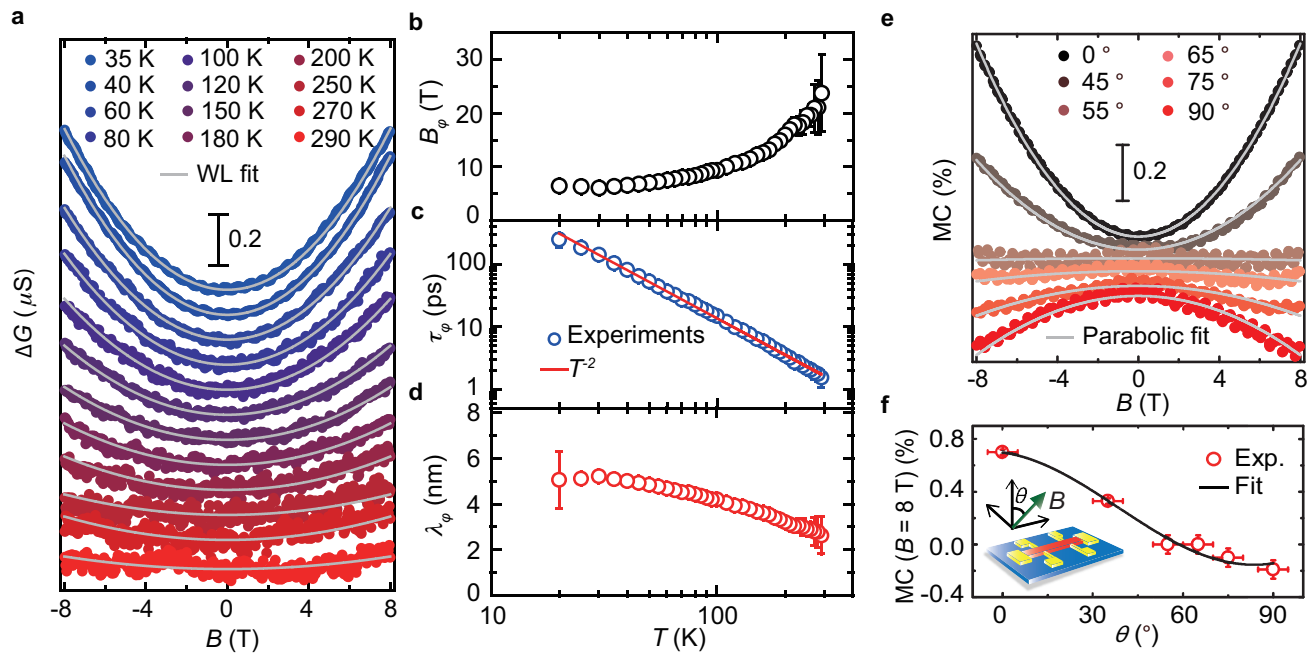

Figure 3: Weak localisation in the magnetoconductance of doped PBTTT/F F $_{4}$ TCNQ.

a, $B$ dependence of differential conductance $(\Delta G(B)=G(B)-G(0))$ at various temperatures; $B$ was applied perpendicular to the substrate plane. The solid curves represent fits based on twodimensional HLN model. b-d, The $T$ dependence of the extracted $B_{\varphi}(\mathbf{b})$, the phase coherence time, $\tau_{\varphi}(\mathbf{c})$, the phase coherence length, $\lambda_{\varphi}(\mathbf{d})$. e, Anisotropy of the magnetocondutance ratio, $M C(B)=\Delta G(B) / G(0)(\%)$ measured at $35 \mathrm{~K}$ for different angle of the magnetic field with the substrate normal. The solid curves are parabolic fits. f, Angular dependence of MC at $8 \mathrm{~T}$ together with a $\cos ^{2}(\theta)$ fit. The out-of-plane angle, $\theta$, is defined in the inset. 

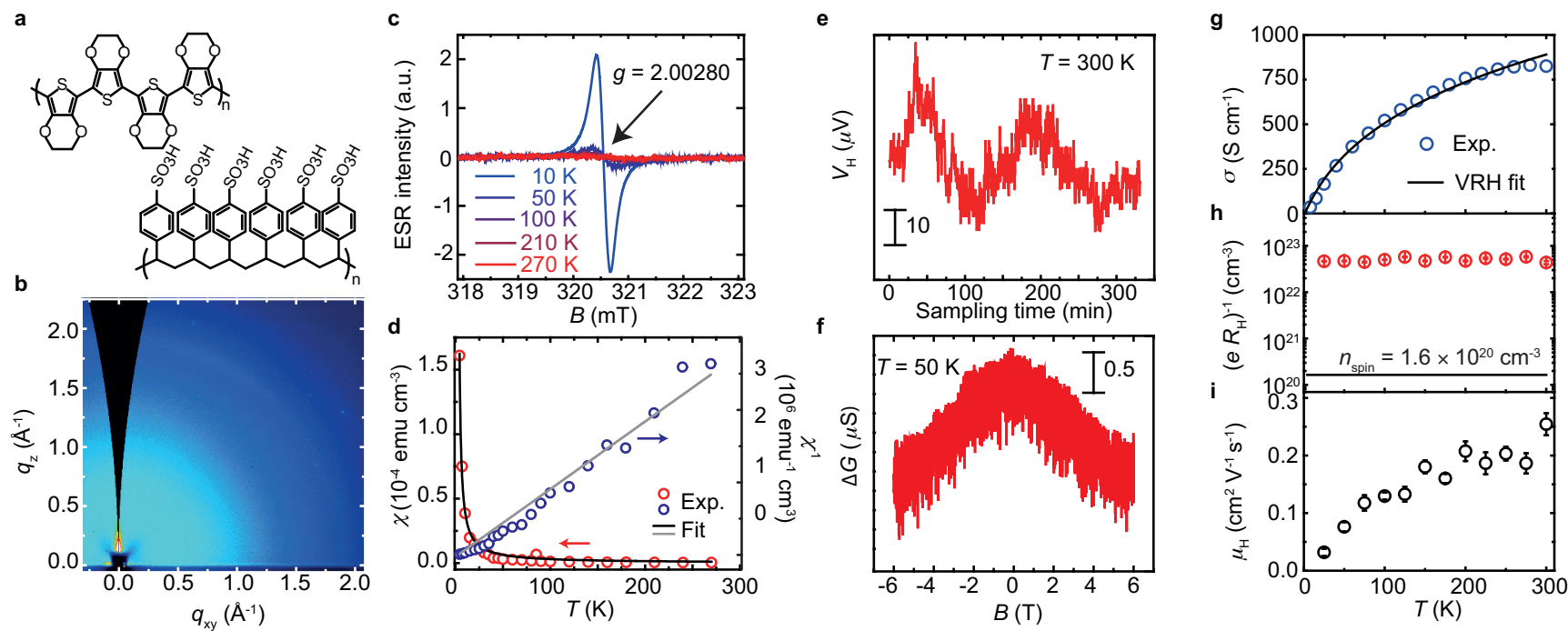

Figure 4: Comparison with the transport properties of disordered PEDOT:PSS. a, Chemical structure of PEDOT:PSS. b, GIWAXS image of PEDOT:PSS. $\mathbf{c}$, ESR spectra measured for PEDOT:PSS at different temperatures. $\mathbf{d}, T$ dependence of $\chi$ plotted in red points, where the black curve represents the $T^{-1}$ dependence, expected from the Curie susceptibility. The same data are plotted in blue points as $\chi^{-1}-T$ plot and the fit from the Curie law in grey line. e, Hall voltage, $V_{\mathrm{H}}$, as a function of sampling time, measured at $300 \mathrm{~K}$; the scale bar represents $10 \mu \mathrm{V}$. f, $\Delta G(B)$ measured at $50 \mathrm{~K}$; the scale bar represents $0.5 \mu \mathrm{S} . \mathbf{g}, T$ dependence of the conductivity $(\sigma)$, where the black curve represents a fitting result for 3D VRH. h, $T$ dependence of the carrier concentrations where the red circles denote the charge concentration $n$ estimated from $R_{\mathrm{H}}^{-1}$. The solid line denotes the spin concentration $n_{\text {spin }}$ estimated from the Curie component of the ESR measurement (Fig. 4c). i, $T$ dependence of the $\mu_{\mathrm{H}}$ estimated from $\mu_{\mathrm{H}}=R_{\mathrm{H}} \sigma$ with a Hall factor $\left(\gamma_{\mathrm{H}}\right)$ of unity. The errors were dominantly from the uncertainty in $R_{\mathrm{H}}$ from Hall voltage. 